SRC TR $88-13$

On Statics of Elastic Systems and Networks of Rigid Bodies

by

J. Loncaric 


\title{
On Statics of Elastic Systems and Networks of Rigid Bodies
}

\author{
J. Lonðarić ${ }^{*}$ \\ University of Maryland ${ }^{\dagger}$
}

October 28,1987

\begin{abstract}
Elastic networks consist of rigid bodies interconnected with generalized springs. Allowing more general interactions leads to a theory of rigid body systems. Stiffness of an elastic system is defined and computed. Compactification of the space of such systems is conveniently interpreted in a grassmannian framework as the set of all Lagrangian planes in the displacement-force space. This compactified space consists of systems which may be constrained by holonomic constraints.
\end{abstract}

\section{Introduction}

It is possible and desirable to develop a theory of rigid bodies interacting via generalized springs. There are examples of similar past efforts ([Whi82], [MS85], [CK87]) in robotics. The problem is to determine the elastic forces which arise when manipulated objects are brought into contact. The lumped model of this situation consists of rigid bodies representing objects interconnected by springs. A potential energy function describes the response of the system.

*This work was supported in part by the Army Research Office grant DAGG-83-K-0027, by ARO/DARPA grant DAAG29-85-K-0096, and by the National Science Foundation under MEA-83-18972.

'Author's present address is Systems Research Center, University of Maryland, College Park, MD 20742, U.S.A. 
The theory developed here is based on Lie groups and differential geometry, following methodology developed in [Arn78] and [AM78]. There are conceptual benefits associated with this approach resulting from the clearly outlined mathematical structure of the problem. Some important geometric features of elastic networks with holonomic constraints will be pointed out.

Throughout this paper an effort has been made to avoid basing the problem description on a specific choice of coordinates. This makes it easy to choose coordinates adapted to the problem later on.

\subsection{Preliminaries}

The physical space is given an Euclidean metric by a choice of length scale and orientation ${ }^{1}$. This object will be denoted by $E^{3}$ and a distinction will be made between $E^{3}$ and $\Re^{3}$ for the following reasons:

- $E^{3}$ is not a vector space whereas $\Re^{3}$ is.

- $\mathrm{E}^{3}$ is isotropic whereas $\mathfrak{R}^{3}=\left\{\left(x_{1}, x_{2}, x_{3}\right)\right\}$ has prefered directions along $x_{i}, i=1,2,3$.

- $E^{3}$ is homogeneous whereas $\Re^{3}$ has a distinguished point - the origin.

- It is desirable to use several ways of identifying $\mathrm{E}^{3}$ with $\Re^{3}$ after the problem has been described.

The coordinate maps $x: E^{3} \longrightarrow \Re^{3}$ are required to preserve the chosen Euclidean metric as well as orientation. There is a good way of visualizing the coordinate map $x$ as a coordinate frame. The codomain $\Re^{3}$ of $x$ has a distinguished point (the origin) and distinguished unit vectors which point along the three axes of $\Re^{3}$. The inverse image of those features of $\Re^{3}$ defines a frame of $x$ in $\mathrm{E}^{3}$, and this frame uniquely determines $x$.

This paper treats rigid bodies as open three dimensional submanifolds of $E^{3}$. Their motions preserve the chosen metric and orientation. One can easily show that a motion of a rigid body uniquelly extends to a rigid

\footnotetext{
${ }^{1}$ Length scale is chosen by using meters, centimeters, feet or some other unit of length. Orientation can be left or right handed. One usually works with right handed orientation.
} 
motion of $E^{3}$. It is well known that the group of such motions is $\operatorname{SE}(3)$, the special euclidean group in three dimensions.

The elements of SE(3) are maps $g: E^{3} \longrightarrow E^{3}$ and the commutative diagram

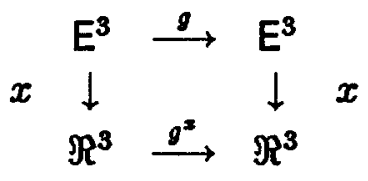

defines the numerical representation of $g$ in terms of a specific choice of coordinates $x$. Since $g^{x}=x \circ g \circ x^{-1}$, changing coordinates to $y$ gives

$$
g^{y}=y \circ g \circ y^{-1}=y \circ x^{-1} \circ g^{x} \circ x \circ y^{-1}
$$

and it is seen that changes of coordinates induce conjugation by $y \circ x^{-1}$ on the representations of rigid motions. Note that $y \circ x^{-1}$ is in itself a representation of a rigid body motion, which maps the coordinate frame of $y$ to the frame of $x$ when interpreted relative to either $y$ or $x$.

We shall consider a given configuration of $n$ elastically coupled rigid bodies. Since displacement of each body from this initial configuration is given by an element of $\mathrm{SE}(3)$, the configurations of this system are in $\operatorname{SE}(3)^{n}$.

\section{Systems and networks}

This section deals with statics of a system or a network of rigid bodies. We will show that a distinction needs to be made between these two concepts. First, by an elastic system we mean a smooth real valued function

$$
V: \operatorname{SE}(3)^{n} \longrightarrow \Re
$$

and the set of all such elastic systems will be denoted by $S$. This definition allows elastic systems whose potential depends on their position in space even when the relative positions of the rigid bodies remain fixed. An elastic system which is free of any such dependence will be called a free elastic system. Free elastic systems must satisfy

$$
V=V \circ L_{g}
$$


for any left translation $L_{g}$ by a $g \in \operatorname{diag}\left(\operatorname{SE}(3)^{n}\right)$. The diagonal subgroup of a product group is in our case defined by

$$
\operatorname{diag}\left(\operatorname{SE}(3)^{n}\right)=\left\{\left(g_{1}, \ldots, g_{n}\right) \in \operatorname{SE}(3)^{n} \| g_{1}=g_{2}=\ldots=g_{n}\right\} .
$$

On the other hand, we are clearly interested in those special elastic systems which arise as networks of interconnected generalized springs. The set of all elastic networks, $\mathcal{N}$, turns out to be a proper subset of $\mathcal{S}$.

A free elastic network can be represented by a directed graph in which each node is a rigid body and each edge is a generalized spring. Let $I$ be the index set labeling the rigid bodies and $J$ be another index set labeling the generalized springs. The potential energy in the network is given by

$$
V=\sum_{j \in J} r_{j}^{*} V_{j}
$$

where $r_{j}^{*}$ is the pullback of the relative position map associated with the edge $j$, and

$$
V_{j}: \mathrm{SE}(3) \longrightarrow \Re
$$

is the potential function of the generalized spring $j$. If this edge is directed from the rigid body $i_{1}$ to the rigid body $i_{2}$, the map $r_{j}$ is given by

$$
\begin{aligned}
& r_{j}: \operatorname{SE}(3)^{n} \longrightarrow \mathrm{SE}(3) \\
& r_{j}\left(g_{1}, \ldots, g_{n}\right)=g_{i_{2}} g_{i_{1}}^{-1}
\end{aligned}
$$

and the pullback $r_{j}^{*} V_{j}=V_{j} \circ r_{j}$.

A general elastic network need not be free, since we can have springs connecting its rigid bodies to the reference rigid body. This additional body does not move, but it can absorb arbitrary forces, and it can be thought of as the ground. If a spring $j$ is directed from the reference body to body $i$, its contribution to the potential is $r_{j}^{*} V_{j}$ where $r_{j}=\pi_{i}$ is the projection from the product group $\operatorname{SE}(3)^{n}$ to its $i$-th component. We will always label the reference rigid body by 0 , so that the index set $I$ is enlarged to $I_{0}=I \cup\{0\}$.

\subsection{Equilibrium and stiffness}

The equilibrium of an elastic system is characterized by $d V=0$ and the stability of such equilibrium can in most cases be decided from the spectrum 
of the Hessian $d^{2} V$ at that point. For elastic networks, the situation is analogous but slightly more complicated. While

$$
d V=\sum_{j \in J} r_{j}^{*} d V_{j}=0
$$

characterizes an equilibrium, clearly it does not imply that individual generalized springs $V_{j}$ are in an equilibrium. In fact, in most interesting situations those springs do exert nonzero forces. Thus, " $d^{2} V_{j}$ " is not a Hessian of $V_{j}$ and we need to define it here. The $d^{2} V_{j}$ shall be represented relative to $q$ by the matrix of second partials

$$
\left(\frac{\partial^{2} V_{j}}{\partial q_{\alpha} \partial q_{\beta}}\right)
$$

where $q: \mathrm{SE}(3) \longrightarrow \Re^{6}$ are coordinates on $\mathrm{SE}(3)$ belonging to the family of exponential coordinates. These exponential coordinates can be easily constructed around the identity in a Lie group as follows. Given a basis of the Lie algebra $s e(3)$ let $q(g)$ be the components of $\exp ^{-1}(g)$ relative to that basis. Clearly changes of coordinates within this family of exponential coordinates are linear, and thus the above matrix behaves tensorially under such changes of coordinates.

\section{Elastic networks}

Let us consider an elastic network which is in equilibrium at its current configuration, i.e. at $e \in \mathrm{SE}(3)^{n}$. The cartesian product structure of the configuration space $\mathrm{SE}(3)^{n}$ leads to a natural notion of displacement and force components, since the tangent and cotangent space at $e$ are direct sums of $n$ copies of $s e(3)$ and $s e(3)^{*}$ respectively. A displacement vector $\gamma \in s e(3)^{n}$ has components $\gamma_{i} \in s e(3)$ where $i \in I$. Similarly, components of a force vector $\Gamma \in \operatorname{se}(3)^{* n}$ are $\Gamma_{i} \in \operatorname{se}(3)^{*}$.

The following propositions, which tell us how to compute $d V$ and $d^{2} V$ from $d V_{j}$ and $d^{2} V_{j}$, are easy to prove. The edge $j$ is assumed to be directed from body $i_{1}$ to body $i_{2}$, where $i_{1}, i_{2} \in I \cup\{0\}$ 
Proposition 3.1 The force $r_{j}^{*} d V_{j}$ has components $\Gamma_{i}$ where $i \in I$ and

$$
\Gamma_{i}= \begin{cases}-d V_{j} & \text { if } i=i_{1} \\ d V_{j} & \text { if } i=i_{2} \\ 0 & \text { otherwise }\end{cases}
$$

This proposition follows from the definition of $r_{j}$ and the properties of the pullback, and it can easily be proven by choosing a matrix representation of $\mathrm{SE}(3)$ and doing explicit calculations. The immediate consequence of this result, which is basically a statement of the action-reaction principle, is

Proposition 3.2 At equilibrium $d V=0$ the sum of all forces exerted on each free body is zero.

which follows from

$$
d V=\sum_{j \in J} r_{j}^{*} d V_{j}=0
$$

Similarly, the contribution of $V_{j}$ to the stiffness of $V$ can be determined. Note that we can think of $d^{2} V$ as a matrix of maps $\mathbf{k}_{a b}: s e(3)_{b} \rightarrow s e(3)_{a}^{*}$, where subscripts indicate the components of the direct sum $s e(3)^{n}$ and its dual.

$$
d^{2} V=\mathbf{K}=\left(\mathbf{k}_{a b}\right) \quad \text { where } a, b \in I
$$

Clearly, the stiffness of the spring $j$ will have nonzero entries $\mathbf{k}_{a b}$ only where $a, b \in\left\{i_{1}, i_{2}\right\} \cap I$. We will denote this submatrix by $\mathbf{K}_{j}$.

Proposition 3.3 The nonzero components of the stiffness $r_{j}^{*} d^{2} V_{j}$ belong to the submatrix $\mathbf{K}_{j}$ which is given by

$$
\mathbf{K}_{j}=\left(\begin{array}{cc}
d^{2} V_{j} & -d^{2} V_{j} \\
-d^{2} V_{j} & d^{2} V_{j}
\end{array}\right)
$$

when both $i_{1}, i_{2} \in I$. When the reference body is involved,

$$
\mathbf{K}_{j}=\left(d^{2} V_{j}\right) .
$$

This proposition gives us a recipe for constructing the stiffness matrix of an elastic network. We begin by choosing a coordinate frame $x$ and expressing all stiffnesses in terms of that fixed frame. 
The diagonal entries of this matrix are sums of stiffnesses of all springs connected to a particular body. Off-diagonal entries $\mathbf{k}_{a b}$ are negative sums of stiffnesses of all springs connecting bodies $a$ and $b$. Note that this implies that $\mathbf{K}$ is not only a symmetric matrix, but also that it is block symmetric, since each $\mathbf{k}_{a b}$ is a symmetric $6 \times 6$ matrix. This clearly demonstrates that although $\mathcal{N} \subset \mathcal{S}, \mathcal{N} \neq S$.

\section{Adapted frames}

A generic spring defines an adapted coordinate frame in terms of which its stiffness is brought to a normal form which maximally decouples rotational and translational aspects of stiffness [Lon85], [Lon88]. An elastic system presents the opportunity to use $n$ coordinate frames. We define a $n$-frame $\hat{x}$ as an $n$-tuple of frames $x_{i}$ where $i \in I$. This coordinate frame induces a particular choice of basis in $s e(3)^{n}$ which determines exponential coordinates in a neighborhood of $e \in \mathrm{SE}(3)^{n}$. The numerical representation $\mathbf{K}^{\hat{x}}$ of coordinate free objects like $\mathbf{K}$ will be denoted by a superscript to indicate which coordinate frame generated it.

Under changes of coordinates $\hat{x} \mapsto \hat{y}=\hat{x} \circ g$ where $g \in \operatorname{SE}(3)^{n}$, the matrix representation of $d^{2} V$ changes from $\mathrm{K}^{2}$ to $\mathbf{K}^{\hat{\theta}}$ as follows:

$$
\mathbf{K}^{\mathfrak{D}}=\operatorname{Ad}_{g}^{* \mathcal{x}} \mathbf{K}^{\underline{x}} \mathrm{Ad}_{g}^{\mathcal{x}}
$$

where $\operatorname{Ad}_{g}$ is the Jacobian of the conjugation map $h \mapsto g h g^{-1}$. The product structure of $\operatorname{SE}(3)^{n}$ shows that $\operatorname{Ad}_{g}$ is a block diagonal map, where the $i$-th block is represented by the familiar $6 \times 6$ matrix

$$
\left(\begin{array}{cc}
R & 0 \\
{[\vec{t}] R} & R
\end{array}\right)
$$

where $R$ is the $3 \times 3$ direction cosine matrix of the $i$-th component of $g$ expressed relative to the $i$-th frame in $\hat{x}$, and $\vec{t}$ is the associated translation component. The notation

$$
[\vec{t}]=\left(\begin{array}{ccc}
0 & -t_{3} & t_{2} \\
t_{3} & 0 & -t_{1} \\
-t_{2} & t_{1} & 0
\end{array}\right)
$$


is used to convert a vector $\vec{t}$ into the antisymmetric matrix which represents the cross product operator $\vec{t} \times$.

We can choose $g$ so that the diagonal blocks maximally decouple rotational and translational aspects of stiffness (see [Lon85] or [Lon88]). The following proposition states this result.

Proposition 4.1 It is possible to find a frame $\hat{y}$ such that the diagonal blocks of $\mathbf{K}^{0}$ are of the form

$$
\mathbf{k}_{i i}^{0}=\left(\begin{array}{cc}
A_{i} & B_{i} \\
B_{i}^{\prime} & C_{i}
\end{array}\right)
$$

where $A_{i}=A_{i}^{\prime}, C_{i}=C_{i}^{\prime}$, and $B_{i}=D_{i}+\left[\overrightarrow{b_{i}}\right]$ are $3 \times 3$ matrices; where $D_{i}$ is a diagonal matrix and $\overrightarrow{b_{i}} \in \operatorname{ker}\left(\operatorname{tr}\left(C_{i}\right) I-C_{i}\right)$.

In a generic case, $\operatorname{tr}\left(C_{i}\right) \notin \sigma\left(C_{i}\right)$, and $B_{i}$ is a pure diagonal matrix. Furthermore, the origin of the $i$-th component frame of $\hat{y}$ is a uniquely determined point in space called the center of stiffness. This situation is discussed in detail in [Lon85] and [Lon88].

\section{$5 \quad$ Building larger elastic systems}

The set of all elastic systems of order $n$ forms a vector space. Given two elastic systems $V, V^{\prime}$ on $n$ rigid bodies, we can construct a larger one by adding the potential functions. The composite system $W=V+V^{\prime}$ is very interesting, since it naturally arises as the result of a parallel as well as a serial connection of springs.

Example 5.1 Consider two bodies connected by a spring. We wish to connect another spring in parallel. The potential of the two springs is simply the sum of their individual potentials.

Example 5.2 Consider two springs connected in series between bodies a to $b$ to $c$. The potential of this system is the sum of the potentials of the spring from $a$ to $b$ and the spring from $b$ to $c$. 
We will work out the later example in detail. There are three rigid bodies, so the configuration space is $\operatorname{SE}(3)^{3}$. We will use $I=\{a, b, c\}$ as our index set for the bodies, and $J=\{1,2\}$ as the index set for the springs. The first spring will be a directed edge from $a$ to $b$, while the second spring will connect $b$ to $c$. The potential function of the first spring is $V=r_{1}^{*} V_{1}$ while the second spring has potential $V^{\prime}=r_{2}^{*} V_{2}$. The composite system has potential function $W=V+V^{\prime}$, which can be explicitly written as a function of $g=\left(g_{a}, g_{b}, g_{c}\right)^{\prime}$ as

$$
W\left(g_{a}, g_{b}, g_{c}\right)=V_{1}\left(g_{a}^{-1} g_{b}\right)+V_{2}\left(g_{b}^{-1} g_{c}\right) \text {. }
$$

The force exerted on this system in order to keep it in the current configuration is $\Gamma=\left(\Gamma_{a}, \Gamma_{b}, \Gamma_{c}\right)=d W$ or

$$
\left(\Gamma_{a}, \Gamma_{b}, \Gamma_{c}\right)=\left(-d V_{1}, d V_{1}-d V_{2}, d V_{2}\right)
$$

while the stiffness of this system is a matrix of maps $s e 3 \rightarrow s e(3)^{*}$ given by

$$
\left(\begin{array}{ccc}
d^{2} V_{1} & -d^{2} V_{1} & 0 \\
-d^{2} V_{1} & d^{2} V_{1}+d^{2} V_{2} & -d^{2} V_{2} \\
0 & -d^{2} V_{2} & d^{2} V_{2}
\end{array}\right): s e(3)^{3} \longrightarrow s e(3)^{* 3}
$$

At an equilibrium, $\Gamma=0$ and therefore $d V_{1}=d V_{2}=0$. We can, however, imagine a slightly more complicated situation, where there is another spring number 3 connecting $a$ to $c$. This gives a new system $W^{\prime}$ whose potential is

$$
W^{\prime}\left(g_{a}, g_{b}, g_{c}\right)=V_{1}\left(g_{a}^{-1} g_{b}\right)+V_{2}\left(g_{b}^{-1} g_{c}\right)+V_{3}\left(g_{a}^{-1} g_{c}\right)
$$

giving the following expression for the force

$$
\Gamma^{\prime}=\left(-d V_{1}-d V_{3}, d V_{1}-d V_{2}, d V_{3}+d V_{2}\right)
$$

and stiffness

$$
\left(\begin{array}{ccc}
d^{2} V_{1}+d^{2} V_{3} & -d^{2} V_{1} & -d^{2} V_{3} \\
-d^{2} V_{1} & d^{2} V_{1}+d^{2} V_{2} & -d^{2} V_{2} \\
-d_{3}^{V} & -d^{2} V_{2} & d^{2} V_{2}+d^{2} V_{3}
\end{array}\right)
$$

This modified system need not have $d V_{j}=0$ at an equilibrium since internal forces can be present. 


\subsection{Vector space of stiffnesses}

The vector space of elastic systems is infinite dimensional, but the stiffnesses form a finite dimensional vector space. The dimension of this vector space can be determined for each family of such systems as a function of the number of bodies involved. Let us denote by $S$ and $\mathcal{N}$ the spaces of elastic systems and networks respectively, and their free versions by $S_{f}$ and $\mathcal{N}_{f}$.

Proposition 5.1 The dimension of the vector space of stiffnesses is given by:

$$
\begin{array}{cl}
\frac{6 n(n-1)}{2} & \text { for systems in } S \\
\frac{6(n-1)(6(n-1)-1)}{2} & \text { for systems in } S_{f} \\
\frac{21 n(n-1)}{2} & \text { for systems in } \mathcal{N} \\
\frac{21(n-1)(n-2)}{2} & \text { for systems in } \mathcal{N}_{f}
\end{array}
$$

The proof of this proposition comes from the observations that the elastic systems have $6 n \times 6 n$ symmetric stiffness matrices, that elastic networks have $n \times n$ block symmetric stiffnesses where each $6 \times 6$ block is symmetric; and that each free system or network corresponds to a non-free version with one less body.

\section{Linearized stability analysis}

Although this section does not discuss dynamics, we will assume that the energy is slowly dissipated by the system. If a small amount of energy is added to the system at an equilibrium, it is certain to settle back to that equilibrium when the potential energy forms a sufficiently deep well. Thus, we will say that an elastic system $V$ is linearly stable when the stiffness $d^{2} V$ is positive definite.

Obviously, free elastic systems are never linearly stable, since they cannot resist left translations by those $g$ which move each body the same way. Both of our examples are free systems and therefore not linearly stable. However, if we modify the system $W^{\prime}$ so that the body $a$ is the reference 
body, we get a second order system $U: \operatorname{SE}(3)^{2} \longrightarrow \Re$ where

$$
U\left(g_{b}, g_{c}\right)=V_{1}\left(g_{b}\right)+V_{2}\left(g_{b}^{-1} g_{c}\right)+V_{3}\left(g_{c}\right)
$$

giving the following expression for the force

$$
\Gamma=\left(d V_{1}-d V_{2}, d V_{3}+d V_{2}\right)
$$

and stiffness

$$
\left(\begin{array}{cc}
d^{2} V_{1}+d^{2} V_{2} & -d^{2} V_{2} \\
-d^{2} V_{2} & d^{2} V_{2}+d^{2} V_{3}
\end{array}\right)
$$

This stiffness matrix can represent a serial connection of springs between the reference body and the body $c$. Suppose $V_{3}$ represents a very stiff positioning device which is holding the body $c$ fixed relative to the ground. The linear stability of this system will then depend on the internal stability of the body $b$. This internal stability results when $d^{2} V_{1}+d^{2} V_{2}$ is positive definite. Thus, we can define that a series connection of springs is internally stable if and only if their parallel connection is stable.

A stable elastic systems can alternatively be represented by the compliance matrix which is obtained by inverting the stiffness matrix. We shall, however, pursue another approach which proves particularly fruitful since it generalizes both concepts.

\section{Stiffness graph}

Given a spring whose stiffness is $\mathbf{K}$, we will consider the graph of $\mathbf{K}$ defined by

$$
G(\mathbf{K})=\left\{(\gamma, \Gamma) \in \operatorname{se}(3)^{n} \oplus \operatorname{se}(3)^{* n} \mid \Gamma=\mathbf{K} \gamma\right\}
$$

The graph of $K$ is a $6 n$-dimensional hyperplane in a $12 n$-dimensional vector space. In general, a space of $k$-planes in $m$-space is called a Grassmannian and denoted by $\operatorname{Grass}(k, m)$. Therefore, the graphs $G(\mathbf{K})$ are elements of Grass $(6 n, 12 n)$.

The linear maps $\mathbf{K}$ are bounded, which implies that their graphs $G(\mathbf{K})$ form a non-compact subset of $\operatorname{Grass}(6 n, 12 n)$. In this section, let us denote by $G(S)$ the set of all points in Grass $(6 n, 12 n)$ obtained as a graph of some system in $S$. The closure $\overline{G(S)}$ includes some additional hyperplanes 
which can be obtained as limits of very stiff springs. We have the following proposition:

Proposition 7.1 An elastic system $V$ of order $n$ subject to $k$ locally holonomic constraints $C_{\kappa}(g)=0, \kappa=1, \ldots, k$ where $C: \operatorname{SE}(3)^{n} \longrightarrow \Re^{k}$ and where $C(e)=0$ is characterized by the hyperplane

$$
G\left(\left.d^{2} V\right|_{\operatorname{ker}(d C)}\right) \oplus \operatorname{span}(d C)
$$

Corollary 7.1.1 The elastic system $V=0$ subject to holonomic constraints $C$ is characterized by the hyperplane

$$
\operatorname{ker}(d C) \oplus \operatorname{span}(d C) \quad
$$

The above corollary follows immediately from the preceeding proposition, which we shall prove here. Recall that $d C$ is a $k$-vector of 1 -forms, whose kernel consists of those vectors in $s e(3)^{n}$ which preserve the identity $C(g)=0$. The span of $d C$, which we will call the internal force space, consists of all linear combinations of the 1 -forms $d C_{\kappa}$. If we model the constraint $C$ as an infinitely stiff spring, we see that it can support any force in $\operatorname{span}(d C)$ with vanishingly small displacement. We shall consider all points $(\gamma, \Gamma) \in s e(3)^{n} \oplus s e(3)^{* n}$ which are compatible with the stiffness $\mathbf{K}=d^{2} \vec{V}$ and the constraints $C$.

First, notice that $d C(\gamma)=0$ if the constraints are to be satisfied and therefore $\gamma \in \operatorname{ker}(d C)$. Thus, our hyperplane has to lie in $\operatorname{ker}(d C) \oplus s e(3)^{* n}$. Let us consider a point $\gamma \in \operatorname{ker}(d C)$ and find all $\Gamma \in s e(3)^{* n}$ which are compatible with $\gamma$. Clearly $\Gamma=\mathbf{K}(\gamma)$ is compatible. On the other hand, the constraint $C$ allows for any internal force to be added to that $\Gamma$. Thus, the system is characterized by a hyperplane which is a direct sum of the graph of $\mathbf{K}$ restricted to $\operatorname{ker}(d C)$ and the span of $d C$, and our proof is complete.

Another result of interest to us explains what happens when two elastic systems with constraints are connected in parallel, so that their stiffnesses add and both constraints have to be satisfied.

Proposition 7.2 Given two systems $S_{1}, S_{2} \in \overline{G(S)}$ characterized by

$$
\begin{aligned}
& S_{1}=G\left(\left.d^{2} V_{1}\right|_{\operatorname{ker}\left(d C_{1}\right)}\right) \oplus \operatorname{span}\left(d C_{1}\right) \\
& S_{2}=G\left(\left.d^{2} V_{2}\right|_{\operatorname{ker}\left(d C_{2}\right)}\right) \oplus \operatorname{span}\left(d C_{2}\right)
\end{aligned}
$$


their parallel connection is characterized by

$$
G\left(\left.d^{2}\left(V_{1}+V_{2}\right)\right|_{\operatorname{ker}\left(d C_{1}\right) \cap \operatorname{ker}\left(d C_{2}\right)}\right) \oplus\left(\operatorname{span}\left(d C_{1}\right) \oplus \operatorname{span}\left(d C_{2}\right)\right)
$$

This proposition follows from the fact that the resulting system has potential function $V_{1}+V_{2}$ and that the space of free directions is obtained by intersection. Note that this result tells us how to build (in stages) representations of complicated elastic systems with multiple constraints. In particular, we now know how to work with elastic networks with networks of constraints. This completes the development of a formalism which enables us to regard stiffness, compliance, and constraints as different views of the same object, i.e. a point in $\operatorname{Grass}(6 n, 12 n)$.

\section{Lagrangian planes and reciprocity}

There is a natural antisymmetric form $\omega$ in $\operatorname{se}(3)^{n} \oplus \operatorname{se}(3)^{* n}$ given by

$$
\left\langle\left(\gamma_{1}, \Gamma_{1}\right),\left(\gamma_{2}, \Gamma_{2}\right)\right\rangle_{\omega}=\Gamma_{1}\left(\gamma_{2}\right)-\Gamma_{2}\left(\gamma_{1}\right)
$$

where $\gamma_{\lambda} \in \operatorname{se}(3)^{n}$ while $\Gamma_{\lambda} \in \operatorname{se}(3)^{* n}$. A plane in $\operatorname{Grass}(6 n, 12 n)$ is reciprocal if $\omega$ is zero for all vectors in that plane. The Betti reciprocity theorem [MH83] applied to our situation states that any plane in $G(S)$ is reciprocal. By continuity of the form $\omega$ we can conclude that all planes in $\overline{G(S)}$ are reciprocal. Furthermore, the converse is also true: a reciprocal plane in $\operatorname{Grass}(6 n, 12 n)$ is in $\overline{G(S)}$. To see that, one need only show that reciprocity is basically equivalent to the fact that stiffnesses are symmetric matrices.

Some standard results [Arn78], [AM78] on symplectic geometry can be applied to the symplectic vector space $\left(s e(3)^{n} \oplus s e(3)^{* n}, \omega\right)$. The null planes (which are self $\omega$-orthogonal, and also called isotropic) of dimension $6 n$ are called Lagrangian planes. The Lagrangian planes are the highest dimensional null planes. We have just shown that the set of elastic systems with constraints corresponds to the set of Lagrangian planes.

In [Arn78] it is shown that every Lagrangian plane is transversal to at least one of the $2^{6 n}$ coordinate Lagrangian planes. These coordinate planes are obtained by choosing a basis for $s e(3)^{n}$ and then taking the span of $6 n$ vectors in $s e(3)^{n} \oplus s e(3)^{* n}$ where the vector $i$ is either the basis vector $i$ or 
the corresponding dual basis vector. We shall use this result in the next section.

\section{Input-output characterization}

Today's robots are mostly position controlled devices, and static forces which arise during their operation can often be determined from the stiffness matrix. This approach becomes ill-posed when constraints are involved. The compliance matrix approach has similar difficulties dealing with situations in which there are no restoring forces. Nevertheless, one can always consider an elastic system with constraints as an input-output system provided the following definition is adopted:

Definition 9.1 An input-output system is a triple $(\Delta, \Omega, \Sigma)$ of points in Grass $(6 n, 12 n)$ such that

1. $\Sigma \in \overline{G(S)}$ is a graph which characterizes an elastic system with constraints,

2. $\Sigma$ and $\Delta$ are transversal to $\Omega$.

The 6 -hyperplane $\Delta$ is called the input space, while $\Omega$ is the output space. The hyperplane $\Sigma$ is the graph of the input-output map of the system.

This definition clarifies our intuitive notion of an elastic input-output system with constraints. When a rigid body is manipulated by a robot, $n=1$ and joint angles of the robot naturally define a basis of $\operatorname{se}(3)^{n}$ (assuming that the Jacobian of the forward kinematic map is nonsingular). In a position controlled situation, one would like to consider $s e(3)^{n}$ as the input space and $s e(3)^{* n}$ as the output space, but this choice runs into problems as soon as constraints are introduced. This difficulty was solved in [RC83] by choosing a new input-output representation in which some positional basis vectors have been replaced by the corresponding force basis vectors.

Proposition 9.1 Given a basis for se $(3)^{n}$, and a Lagrangian plane $\Sigma$ which characterizes an elastic system with constraints, it is always possible to construct an input-output representation $(\Delta, \Omega, \Sigma)$ such that $\Delta$ and $\Omega$ are coordinate Lagrangian planes. 
This proposition is an immediate result of the application of the theorem on coordinate Lagrangian planes cited from [Arn78] at the end of the previous section. We have thus proven that the hybrid position-force control strategy outlined in [RC83] can always be designed in a well posed way.

Stability of a system controlled by a hybrid position-force controller can also be achieved by this method. The controller is an elastic system characterized by a plane $\Lambda \in \overline{G(S)}$ which is connected in parallel to the system $\Sigma$. The plane $\Lambda$ represents the $m$ positional constraints $P=0$ where $P(g)=\left(q_{i_{1}}, q_{i_{2}}, \ldots, q_{i_{m}}\right)$ and $q_{i}$ are the exponential coordinates obtained from the given basis of $s e(3)^{n}$. Therefore,

$$
\Lambda=\operatorname{ker}(d P) \oplus \operatorname{span}(d P)
$$

and since

$$
\Sigma=G\left(\left.d^{2} V\right|_{\operatorname{ker}(d C)}\right) \oplus \operatorname{span}(d C)
$$

the controlled system is characterized by

$$
G\left(\left.d^{2} V\right|_{\operatorname{ker}(d P) \cap \operatorname{ker}(d C)}\right) \oplus(\operatorname{span}(d P) \oplus \operatorname{span}(d C))
$$

which is certainly stable if $\operatorname{ker}(d P) \cap \operatorname{ker}(d C)=\{0\}$.

Proposition 9.2 Given a basis of se(3) ${ }^{n}$ and an elastic system with constraints $\Sigma \in \overline{G(S)}$ one can construct a stable hybrid position-force controller.

To prove this, note that $\operatorname{span}(d C)$ is transversal (as a $k$-dimensional subspace) to at least one of $6 n-k$ dimensional coordinate planes in $s e(3)^{* n}$, say plane $Z^{*}$. Let $Y^{*}$ be the complement coordinate plane in $s e(3)^{* n}$ so that $Y^{*} \oplus Z^{*}=s e(3)^{* n}$. The basis vectors which span $Y^{*}$ are dual to the basis vectors in $s e(3)^{n}$ whose span is $Y$. Then, the hybrid controller $\Lambda=Y \oplus Z^{*}$ is stable, since $\operatorname{span}(d C) \oplus Z^{*}=s e(3)^{* n}$ and the space of free directions is zero.

The controller $\Lambda$ is a coordinate Lagrangian plane which is transversal to $\Sigma$ and we can use it as the output space. Similarly, $Y^{*} \oplus Z$ is another coordinate Lagrangian plane which is transversal to $\Lambda$, and we can think of it as the input space. 


\section{Conclusions}

We have shown how statics of a system of $n$ rigid bodies with constraints can be described by a Lagrangian plane in the displacement-force space $s e(3)^{n} \oplus \operatorname{se}(3)^{* n}$. The theory developed here can guide the development of computer programs capable of analyzing of such systems. The coordinate independent description of the general problem can easily be expressed in terms of any given choice of basis vectors in $s e(3)^{n}$, and in a generic case, a particular adapted frame can be found. We have also shown how to construct a stable hybrid control strategy which guarantees a valid inputoutput description of a given elastic system with constraints.

\section{References}

[AM78] R. Abraham and J. E. Marsden.

Foundations of Mechanics.

The Benjamin/Cummings Publishing Co., Reading, Mass., 1978.

[Arn78] V. I. Arnold.

Mathematical Methods of Classical Mechanics.

Volume 60 of GTM, Springer-Verlag, New York, 1978.

[CK87] M. R. Cutkosky and I. Kao.

Computing and Controlling the Compliance of a Robotic Hand.

Technical Report 8-31-87, CDR, August 1987.

Submitted to the IEEE Journal of Robotics and Automation.

[Lon85] J. Loncarić.

Geometrical Analysis of Compliant Mechanisms in Robotics.

Ph.D. dissertation, Division of Applied Sciences, Harvard University, June 1985.

[Lon88] J. Lončarić.

Normal forms of stiffness and compliance matrices.

IEEE Journal of Robotics and Automation, to appear:?, ? 1988.

[MH83] J. E. Marsden and T. J. R. Hughes.

Mathematical Foundations of Elasticity.

Prentice-Hall, Inc., Engelwood Cliffs, N.J., 1983. 
[MS85] M. T. Mason and J. K. Salisbury.

Robot Hands and the Mechanics of Manipulation.

MIT Press, Cambridge, Mass., 1985.

[RC83] M. H. Raibert and J. J. Craig.

Hybrid position/force control of manipulators.

In Brady et al., editor, Robot Motion: Planning and Control, The MIT Press, Cambridge, Mass., 1983.

[Whi82] D. E. Whitney.

Quasi-static assembly of compliantly supported rigid parts.

Journal of Dynamic Systems, Measurment, and Control, 104:6577, March 1982. 